\title{
Hadron Production at Fixed Target Energies and Extensive Air Showers
}

\section{Unger* for the NA61/SHINE Collaboration}

Karlsruher Institut für Technologie

E-mail: Michael. Unger@kit.edu

\begin{abstract}
NA61/SHINE is a fixed-target experiment to study hadron production in hadron-nucleus and nucleus-nucleus collisions at the CERN SPS. Due to the very good acceptance and particle identification in forward direction, NA61/SHINE is well suited for measuring particle production to improve the reliability of air shower simulations. Data with proton and pion beams have been taken in 2007 and 2009. First analysis results for the pion yield in proton-carbon interactions at $30 \mathrm{GeV}$ will be shown and compared to predictions from models used in air shower simulations.
\end{abstract}

35th International Conference of High Energy Physics - ICHEP2010,

July 22-28, 2010

Paris France

* Speaker. 


\section{Introduction}

Ultra-high energy cosmic rays initiate extensive air showers (EAS) when they collide with the nuclei of the atmosphere. The interpretation of EAS data as for instance recorded by the Pierre Auger Observatory [3] or the KASCADE air shower array [4] relies to a large extent on the understanding of these air showers and specifically on the correct modeling of hadron-air interactions that occur during the shower development. The relevant particle energies span a wide range from primary energies of $\gtrsim 10^{20} \mathrm{eV}$ down to energies of $10^{9} \mathrm{eV}$. The mesons that decay to muons at ground level typically originate from low energy interactions in the late stages of an air shower. Depending on the primary energy and detection distance, the corresponding interaction energies are between 10 and $1000 \mathrm{GeV}$ (cf. Fig 1). As it has been noted in e.g. [5-8], the modeling of low energy interactions contribute at least $10 \%$ to the overall uncertainty of the predicted muon number at ground. Since these energies are within the reach of fixed target experiments, precise measurements of hadronic particle production at, for instance, the Super Proton Synchrotron at CERN can help to diminish the uncertainties of air

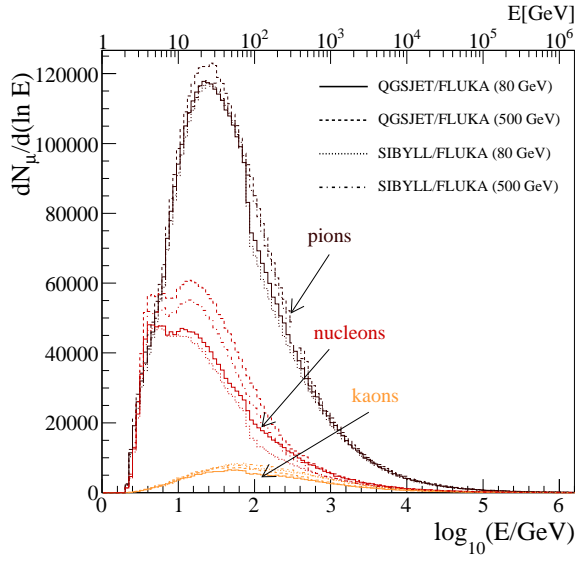

Figure 1: Particle types and energies involved in the last interaction leading to muon detected at ground level $\left(E_{0}=\right.$ $10^{19} \mathrm{eV}$, detection distance $\left.\approx 1 \mathrm{~km}\right)$ [9]. shower simulations.

An example of current difficulties to describe air shower measurements at ultra-high energies is the excess of the number of ground level muons wrt. to air shower simulations [10-12] as reported by the Pierre Auger Observatory [13]. A solution to this inconsistency was proposed in [14], where it was pointed out that an increased production of baryons and anti-baryons in hadron-air
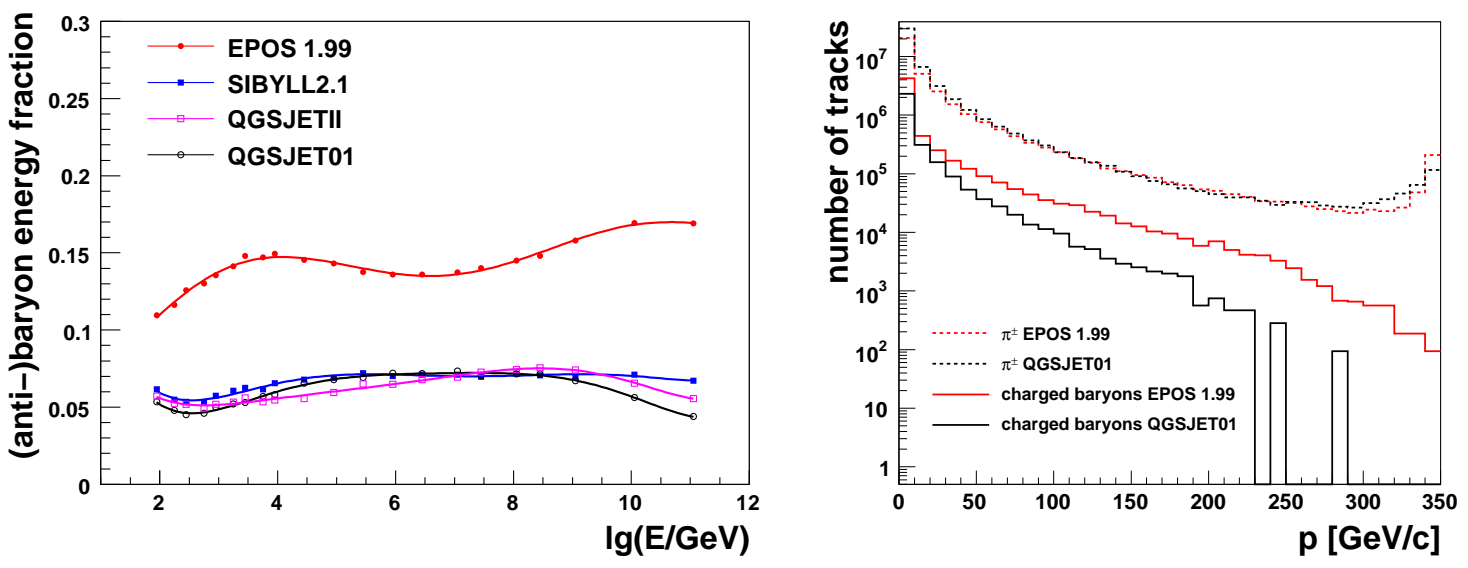

Figure 2: Left panel: Energy fraction of produced baryons and anti-baryons in $\pi$-air collision as a function of pion momentum. Right panel: Expected number of charged tracks as a function of secondary momentum for the NA61 $\pi^{-}-\mathrm{C}$ data set at $350 \mathrm{GeV} / \mathrm{c}$. 
collisions, would lead to an increase in the number of muons at ground level. As can be seen in Fig. 2, an enhanced (anti-)baryon production, as e.g. currently implemented in the EPOS-model, should be easily distinguishable from previous model assumptions at fixed target energies.

Unfortunately, there exist no comprehensive and precise particle production measurements for the most numerous projectile in air showers, the $\pi$-meson. Therefore, new data with pion beams at 158 and $350 \mathrm{GeV} / \mathrm{c}$ on a thin carbon target (as a proxy for nitrogen) has been recently collected by the NA61 experiment at the SPS.

\section{The NA61/SHINE Experiment}

NA61/SHINE (SHINE = SPS Heavy Ion and Neutrino Experiment) [1] is an experiment at the CERN SPS using the upgraded NA49 hadron spectrometer. Among its physics goals are precise hadron production measurements for improving calculations of the neutrino beam flux in the T2K neutrino oscillation experiment [2] as well as for more reliable simulations of cosmic-ray air showers. Moreover, $\mathrm{p}+\mathrm{p}$, $\mathrm{p}+\mathrm{Pb}$ and nucleus+nucleus collisions will be studied extensively to allow for a study of properties of the onset of de-confinement and search for the critical point of strongly interacting matter.

The NA61 detector uses large time-projectionchambers to measure the particle charges and momenta as well as their energy loss for particle type identification. Large scintillator walls provide an estimate of the particle's squared mass from the time-of-flight through the detector. The momentum resolution, $\sigma(1 / p)=\sigma(p) / p^{2}$, is about $10^{-4}(\mathrm{GeV} / \mathrm{c})^{-1}$ at full magnetic field and the tracking efficiency is better than $95 \%$.

NA61 started data taking in 2007. After a pilot run

\begin{tabular}{cccc}
\hline \hline & $\begin{array}{c}\mathrm{p} \\
{[\mathrm{GeV} / \mathrm{c}]}\end{array}$ & year & $\begin{array}{c}N_{\text {int }} \\
{\left[10^{6}\right]}\end{array}$ \\
\hline$\pi^{-}-\mathrm{C}$ & 158 & 2009 & 3.6 \\
$\pi^{-}-\mathrm{C}$ & 350 & 2009 & 4.7 \\
p-C & 31 & 2007 & 0.6 \\
p-C & 31 & 2009 & 4.8 \\
p-p & 13 & 2010 & 0.6 \\
p-p & 20 & 2009 & 1.7 \\
p-p & 30 & 2009 & 2.6 \\
p-p & 40 & 2009 & 4.2 \\
p-p & 80 & 2009 & 3.6 \\
p-p & 158 & 2009 & 2.8 \\
p-p & 158 & 2010 & 43.9 \\
\hline \hline
\end{tabular}

Table 1: Number of thin target interaction triggers collected by NA61.

with proton on carbon at $31 \mathrm{GeV} / \mathrm{c}$, the data acquisition system has been upgraded during 2008 to increase the event processing rate by a factor of $\approx 10$. In the last two years, NA61 took data at beam energies from 13 to $350 \mathrm{GeV}$ with proton and pion projectiles and proton and carbon targets (cf. Tab. 2).

\section{Data Analysis}

In this paper we present preliminary results on the inclusive production of positive and negative pions from $\mathrm{p}+\mathrm{C}$ interactions at $31 \mathrm{GeV} / \mathrm{c}$ recorded during the 2007 pilot run. The pion spectra have been obtained using three independent analysis techniques: Firstly, with the so-called $h^{-}$method all negative hadrons produced in a collision are assumed to be pions and the small contribution of other species is corrected for using simulations. Due to the large contribution from protons, this method can only be applied to determine the $\pi^{-}$spectra. Secondly, with the $\mathrm{d} E / \mathrm{d} x$ method $\pi$-mesons 

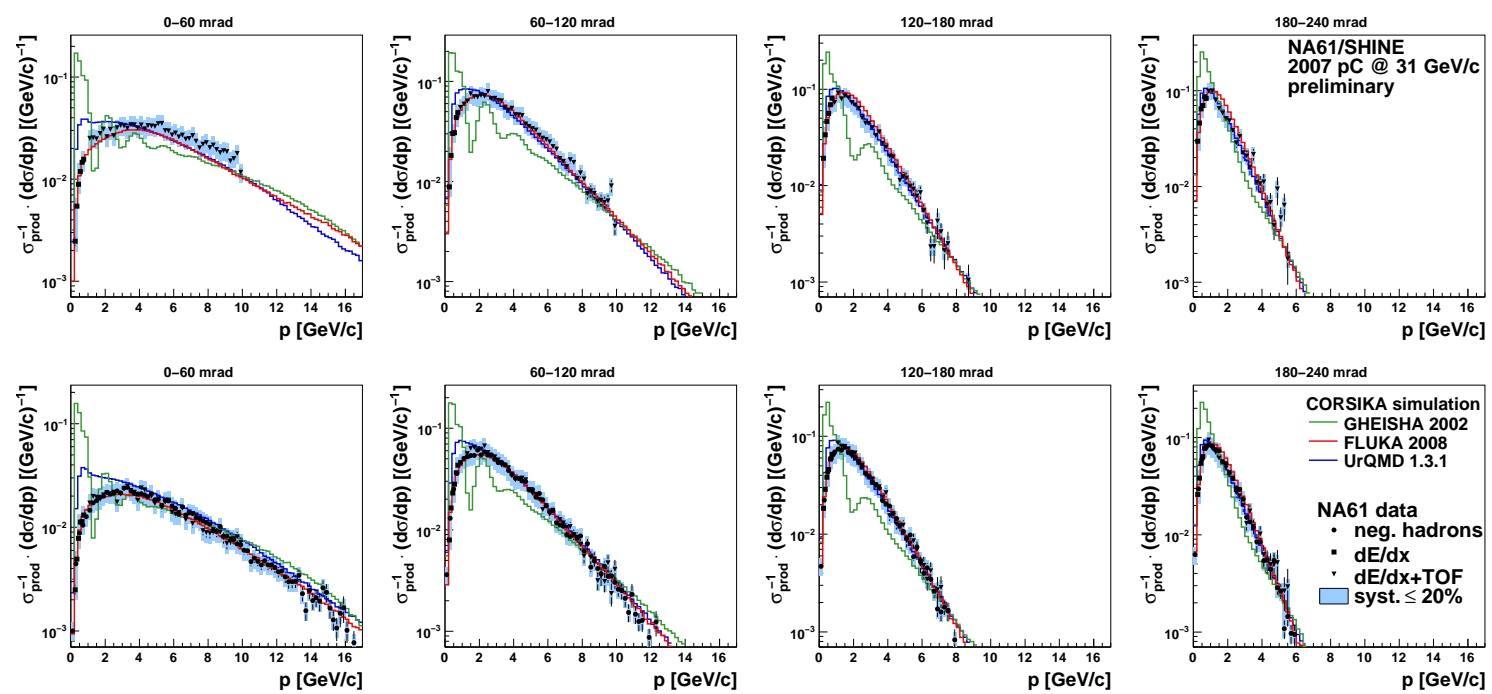

Figure 3: The $\pi^{+}$(upper panel) and $\pi^{-}$(lower panel) spectra in $\mathrm{p}+\mathrm{C}$ interactions at $31 \mathrm{GeV} / \mathrm{c}$ compared to FLUKA2008 (red histogram), GHEISHA2002 (green histogram), and URQMD1.3.1 (blue histogram) models. The spectra are normalized to the total number of inelastic collisions.

are identified explicitly using the energy deposit measured in the TPCs. This method works only in momentum regions where Bethe-Bloch bands do not overlap. Finally, using the $\mathrm{d} E / \mathrm{d} x$-plusToF method, $\pi^{ \pm}$can be identified over a wide momentum range using the combination of energy loss and $m^{2}$ from the time-of-flight measurement. This method provides the most precise particle identification, but it is limited to the angular acceptance of the time-of-flight detectors. Using a combination of all three methods, NA61 is able to measure $\pi^{ \pm}$-spectra with a large acceptance in angle and momentum.

\section{Results}

Preliminary $\pi^{ \pm}$-spectra in $\mathrm{p}+\mathrm{C}$ interactions at $31 \mathrm{GeV} / \mathrm{c}$ from the 2007 pilot run are presented in Fig. 3. The systematic uncertainty of these preliminary spectra is estimated to be $\leq 20 \%$.

As a first application of these measurement, it is interesting to compare the spectra to the predictions of event generators for hadronic interaction. Here we concentrate on hadronic interaction models that have been frequently used for the interpretation of cosmic ray data, i.e. FluKa2008 [15], URQMD1.3.1 [17] and Gheisha2002 [16]. All three models are part of the CORSIKA [18] framework for the simulation of air showers and are typically used to generate hadron-air interactions at energies below $80 \mathrm{GeV}$. To assure that all relevant settings of the generators are identical to the ones used in air shower simulations, we simulated single $\mathrm{p}+\mathrm{C}$ interactions directly with CORSIKA in the so-called interaction test mode. As can be seen in Fig. 3, GHEISHA simulations can not describe our measurements at all production angles and thus this measurement corroborates earlier findings of the short-comings of GHEISHA (see e.g. [8]). The URQMD generator can describe our data better, but fails to reproduce the spectra at low momenta and production angles. The best agreement is found for the spectra generated with FLUKA, that show a good overall agreement with our data. 


\section{Acknowledgments}

This work was supported by the Hungarian Scientific Research Fund (grants OTKA 68506 and 79840), the Polish Ministry of Science and Higher Education (grants 667/N-CERN/2010/0, N N202 1267 36, DWM/57/T2K/2007), the Federal Agency of Education of the Ministry of Education and Science of the Russian Federation (grant RNP 2.2.2.2.1547) and the Russian Foundation for Basic Research (grants 08-02-00018 and 09-02-00664), the Ministry of Education, Culture, Sports, Science and Technology, Japan, Grant-in-Aid for Scientific Research (grants 18071005, 19034011, 19740162), the German Research Foundation (grant GA 1480/2-1), the Institut National de Physique Nucléaire et Physique des Particules (IN2P3, France), Swiss Nationalfonds Foundation (grant 200020-117913/1) and ETH Research Grant TH-01 07-3.

\section{References}

[1] N. Antoniou et al. [NA61/SHINE Collaboration], CERN-SPSC-2007-004, (2007); CERN-SPSC-2007-019, (2007); N. Abgrall et al. [NA61/SHINE Collaboration], CERN-SPSC-2008-018, (2008).

[2] Y. Itow et al. The JHF-Kamiokande neutrino project, [arXiv:hep-ex/0106019], (2001)

[3] J. Abraham et al. [Pierre Auger Collaboration], Nucl. Instrum. Meth. A 523, 50 (2004)

[4] T. Antoni et al. [KASCADE Collaboration], Nucl. Instrum. Meth. A 513, 490 (2003)

[5] C. Meurer et al., Czech. J. Phys. 56 (2006) A211.

[6] H.-J. Drescher et al., Astropart. Phys. 21 (2004) 87.

[7] I. C. Mariş et al., Nucl. Phys. Proc. Suppl. 196 (2009) 86.

[8] D. Heck et al., Proc. 28th ICRC, (2003) 279.

[9] I.C. Mariş et al. [NA61/SHINE Collaboration], Proc. 31st ICRC, (2009).

[10] N.N. Kalmykov and S.S. Ostapchenko, Phys. Atom. Nucl. 56 (1993), 346.

[11] S.S. Ostapchenko, Nucl. Phys. Proc. Suppl. 151 (2006), 143;

[12] E.J. Ahn et al., Phys. Rev. D80 (2009) 094003.

[13] R. Engel et al. [Pierre Auger Collaboration], Proc. 30th ICRC (2007) [arXiv:astro-ph/0706.1921]; A. Castellina et al. [Pierre Auger Collaboration], Proc. 31st ICRC (2009) [arXiv:astro-ph/0906.2319]

[14] T. Pierog and K. Werner, Phys. Rev. Lett. 101 (2008), 171101.

[15] A. Fasso et al., CERN-2005-10 (2005); G. Battistoni et al., AIP Conf. Proc. 896 (2007) 31.

[16] H.Fesefeldt, Aachen PITHA-85-02 (1985); patched by R.E. Cassell and G. Bower (SLAC) (2002).

[17] M. Bleicher et al., J. Phys. G: Nucl. Part. Phys. 25, (1999) 1859.

[18] D. Heck et al., Report Forschungszentrum Karlsruhe FZKA-6019, (1998). 